\title{
ZEB1 promotes tumorigenesis and metastasis in hepatocellular carcinoma by regulating the expression of vimentin
}

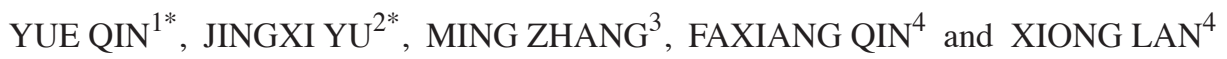 \\ ${ }^{1}$ Department of Physical Examination, Division of Traditional Chinese Medicine, \\ The Central Hospital of Enshi Tujia and Miao Autonomous Prefecture, Enshi, Hubei 445000, P.R. China; \\ ${ }^{2}$ Division of Financial Analysis, University of New South Wales, Kensington, Sydney, NSW 2052, Australia; \\ Departments of ${ }^{3}$ Endocrinology and ${ }^{4}$ Respiratory Medicine, The Central Hospital of Enshi Tujia and \\ Miao Autonomous Prefecture, Enshi, Hubei 445000, P.R. China
}

Received September 5, 2018; Accepted December 12, 2018

DOI: $10.3892 / \mathrm{mmr} .2019 .9866$

\begin{abstract}
Hepatocellular carcinoma (HCC) is one of the most common malignancies worldwide and its prognosis remains poor. Epithelial-to-mesenchymal transition (EMT)-induced markers have emerged as key regulators of tumor development and progression in HCC. The aim of the present study was to investigate the role of zinc finger E-box-binding homeobox 1 (ZEB1) in the tumorigenesis of HCC and to elucidate the mechanism underlying the correlation between ZEB1 and vimentin (VIM). The expression levels of ZEB1 and VIM were assessed by immunohistochemistry, western blotting and reverse transcription-quantitative polymerase chain reaction analysis in HCC tissues and cell lines. The biological significance of ZEB1 was examined by downregulating the expression of ZEB1 in Huh-7 cells. A luciferase reporter assay was used to investigate the association between ZEB1 and VIM. The expression levels of ZEB1 and VIM were higher in tumor tissues compared with those in adjacent normal tissues, and they were significantly associated with a poor prognosis in patients with $\mathrm{HCC}$, whereas ZEB1 silencing led to the attenuation of HCC cell proliferation, invasion and migration. Furthermore, it was observed that ZEB1 was able to bind to a certain site in the VIM promoter and regulate the transcriptional activity of VIM. Therefore, the present study demonstrated that ZEB1 is a potential biomarker of the tumorigenesis and progression of $\mathrm{HCC}$, and it may regulate transcription of the VIM gene.
\end{abstract}

Correspondence to: Dr Xiong Lan, Department of Respiratory Medicine, The Central Hospital of Enshi Tujia and Miao Autonomous Prefecture, 158 Wuyang Road, Enshi, Hubei 445000, P.R. China

E-mail: lanx65@163.com

${ }^{*}$ Contributed equally

Key words: hepatocellular carcinoma, zinc finger E-box-binding homeobox 1, vimentin, regulation, mechanism

\section{Introduction}

Hepatocellular carcinoma (HCC) is one of the most common malignancies worldwide, ranking fifth among all malignant tumors. According to previous reports, it is estimated that $\sim 500,000-1,000,000$ new cases of HCC are diagnosed annually, and $\mathrm{HCC}$ ranks third in terms of mortality rate among all types of cancer (1). Invasion, metastasis and postoperative recurrence are the main causes of mortality in patients with HCC, and they are also the key factors affecting clinical treatment and prognosis. Due to its aggressive course and propensity for metastasis and invasion, HCC is often diagnosed at an advanced stage and is associated with a high mortality rate (2). Previous studies have demonstrated that aberrant activation of epithelial-to-mesenchymal transition (EMT) is important in tumor cell invasion and metastasis. EMT allows normal hepatic epithelial cells to undergo multiple biochemical changes that enable them to assume a mesenchymal phenotype, which includes enhanced migratory capacity, invasiveness, increased resistance to apoptosis and a marked increase in the production of extracellular matrix components (3). It has been gradually realized that EMT involves multiple molecular mechanisms and signal transduction pathways, the hallmark of which is the downregulation of cell adhesion molecule epithelial cadherin (E-cadherin) and the upregulation of mesenchymal molecule vimentin (VIM). In addition, a number of transcriptional factors have been identified as crucial inducers of EMT, including the Snail homologues, the basic helix-loop-helix and the zinc-finger E-box-binding homeobox (ZEB) (4). ZEB1, also referred to as TCF8 or dEF1, is a member of the zinc-finger family of proteins. The zinc finger clusters at both sides of ZEB1 can bind to specific sequences of DNA, thereby regulating the transcription of targeted genes $(5,6)$, which has emerged as a key event in cancer progression. Numerous studies have demonstrated that abnormal expression of ZEB1 in endometrial, colorectal and prostate cancer has been associated with disease aggressiveness, low differentiation, development of metastases and poor prognosis (7-9). It has been demonstrated that, by interacting with the E-box element within the proximal region of the E-cadherin promoter, ZEB1 can affect its expression (10), which is crucial in the occurrence 
and development, and in the metastasis and invasion of most types of cancer (11). However, few studies have examined the correlation between ZEB1 and VIM in HCC, and the specific regulatory mechanism remains to be fully elucidated. The aim of the present study was to investigate the association between the expression of ZEB1 and VIM in HCC tissues and adjacent normal tissues by immunohistochemistry and western blotting. The study also aimed to detect the changes in the biological behavior of Huh7 cells upon downregulation of the expression of ZEB1 by small interfering RNA, in order to evaluate the clinical relevance of the expression status of ZEB1 and VIM and provide data for the prognosis and targeted therapy of HCC.

\section{Patients and methods}

Patients and tumor samples. Fresh surgical resection specimens of HCC and adjacent normal tissues were collected from 80 patients with HCC at The Central Hospital of Enshi Autonomous Prefecture (Enshi, China) from January 2012 to January 2013. Curative resection was defined as the removal of all identifiable tumor tissue with a clear microscopic margin. None of the patients received any preoperative therapy, for example, transcatheter arterial chemoembolization, radiofrequency ablation or percutaneous ethanol injection. The patients included 63 men and 17 women, ranging in age between 33 and 76 years, with a mean age of 56 years. The clinicopathological variables, including the size of the primary tumor, vascular invasion, intrahepatic metastasis, serum $\alpha$-fetoprotein (AFP) levels, liver cirrhosis, differentiation and tumor-necrosis-metastasis (TNM) stage, are listed in Table I. Follow-up data following surgery were obtained from all patients by measurement of the AFP levels and ultrasound or computed tomography at least every 3 months. All patients provided written informed consent to participate in the present study, and the study protocol was approved by the Ethics Committee of The Central Hospital of Enshi Autonomous Prefecture.

Immunohistochemistry. The HCC tissues and the adjacent normal tissues were fixed in $10 \%$ formalin and embedded in paraffin. Serial $4-\mu \mathrm{m}$ sections were cut from each block. Following deparaffinization and rehydration, heat-induced antigen retrieval by autoclave pretreatment $\left(120^{\circ} \mathrm{C}\right.$ for $\left.10 \mathrm{~min}\right)$ in citrate buffer solution ( $\mathrm{pH}$ 6.0) was performed. Endogenous peroxidase activity was deactivated by soaking the sections in absolute methanol solution containing $3 \% \mathrm{H}_{2} \mathrm{O}_{2}$ for $5 \mathrm{~min}$ at room temperature. Subsequently, the sections were treated with $5 \%$ bovine serumalbumin (BeyotimeInstitute of Biotechnology, Shanghai, China) for $30 \mathrm{~min}$ to block non-specific reactions, and were then incubated with anti-ZEB1 antibody (cat. no. ab180905; 1:150 dilution; Abcam, Cambridge, MA, USA) or anti-VIM antibody (cat. no. sc-80975; 1:50 dilution; Santa Cruz Biotechnology, Inc., TX, USA) at $4^{\circ} \mathrm{C}$ overnight and washed with phosphate-buffered saline (PBS). Subsequently, the biotin-labeled secondary antibody (cat. no. ab6789; 1:200 dilution; Abcam) was added, and the sections were incubated at room temperature for $1 \mathrm{~h}$. Following the addition of horseradish peroxidase (HRP)-conjugated streptavidin working solution and washing with PBS, the sections were stained with 3,3'-diaminobenzidine and counterstained with hematoxylin for $15 \mathrm{~min}$, followed by successive steeping in an ethanol solution with hydrochloric acid and dilute ammonia for several minutes. Finally, the sections were dehydrated through gradient alcohol solutions and mounted in neutral balsam. The immunohistochemical staining was evaluated by two experienced pathologists using an inverted microscope. The results were assessed using a double-blind method and were repeated at least three times.

Cell lines and culture. Two human HCC cell lines (Hep3B and Huh-7) and a hepatoblastoma cell line (HepG2) (12) were obtained from American Type Tissue Collection (Manassas, VA, USA). The HCCLM9 cell line was obtained from the Liver Cancer Institute, Fudan University (Shanghai, China) (13). All cells were cultured in RPMI-1640 medium supplemented with $10 \%$ fetal bovine serum (Gibco; Thermo Fisher Scientific, Inc., Waltham, MA, USA) and $1 \%$ penicillin and streptomycin in a humidified atmosphere of $5 \% \mathrm{CO}_{2}$ at $37^{\circ} \mathrm{C}$.

Western blot analysis. Proteins $(20 \mu \mathrm{g})$ from the clinical HCC specimens and cell lines were extracted with lysate buffer (Beyotime Institute of Biotechnology), separated by $7.5 \%$ (for ZEB1) and 10\% (for VIM) SDS-PAGE and transferred onto PVDF membranes; protein concentration was determined via a BCA kit (Beyotime Institute of Biotechnology). Subsequently, the PVDF membrane was blocked with $5 \%$ skimmed milk powder in TBST $[10 \mathrm{mM}$ Tris- $\mathrm{HCl}(\mathrm{pH}$ 7.5), $150 \mathrm{mM} \mathrm{NaCl}$ and $0.1 \%$ Tween-20] at room temperature for $1 \mathrm{~h}$. The anti-ZEB1 antibody (cat. no. sc-515797; 1:200 dilution) and the anti-VIM antibody (cat. no. sc-66001; 1:200 dilution; both Santa Cruz Biotechnology, Inc.) were added and incubated overnight at $4^{\circ} \mathrm{C}$. Following washing with TBST buffer, the HRP-labeled goat secondary antibody (cat. no. ab6789; 1:2,000 dilution; Abcam) was added and the membrane was incubated at room temperature for $1 \mathrm{~h}$. The proteins were visualized by autoradiography using the ECL chemiluminescence reagent (PeptBio, Wuhan, China). The relative expression of the protein of interest was represented as the grayscale ratio of the protein to GAPDH, and the results were analyzed by GraphPad Prism 5.0 software (GraphPad Software, Inc., La Jolla, CA, USA).

Reverse transcription-quantitative polymerase chain reaction $(R T-q P C R)$ analysis. Total RNA was extracted utilizing TRIzol reagent (Invitrogen; Thermo Fisher Scientific, Inc.), according to the manufacturer's protocol. The RNA was reverse transcribed to cDNA using a Takara RNA PCR kit (Takara Bio, Inc, Otsu, Japan). The qPCR procedure was performed with iQ SYBR-Green Supermix (Bio-Rad Laboratories, Inc., Hercules, CA, USA). A reaction volume of $20 \mu \mathrm{l}$ containing $1 \mu \mathrm{l}$ of forward and reverse primers each, $2 \mu \mathrm{l}$ cDNA, $6 \mu \mathrm{lddH_{2 }}$ O and $10 \mu \mathrm{l}$ Supermix. All reactions were run in triplicate on the iCycler IQ multi-color detection system (Bio-Rad Laboratories, Inc.). The amplification profile was denaturation at $95^{\circ} \mathrm{C}$ for $5 \mathrm{~min}$, followed by 40 cycles of denaturation at $95^{\circ} \mathrm{C}$ for $20 \mathrm{sec}$, annealing at $60^{\circ} \mathrm{C}$ for $20 \mathrm{sec}$ and extension at $72^{\circ} \mathrm{C}$ for $20 \mathrm{sec}$. The PCR primers used were as follows: ZEB1 forward, 5'-TGCACTGAGTGTGGAAAA GC-3' and reverse, 5'-TGGTGATGCTGAAAGAGACG-3'; 
Table I. Clinicopathological variables and the expression of ZEB1 and VIM in hepatocellular carcinoma.

\begin{tabular}{|c|c|c|c|c|c|c|c|}
\hline \multirow[b]{2}{*}{ Variable } & \multirow[b]{2}{*}{$\begin{array}{l}\text { Total no. } \\
(\mathrm{n}=80)\end{array}$} & \multicolumn{3}{|c|}{ ZEB1 } & \multicolumn{3}{|c|}{ VIM } \\
\hline & & $\begin{array}{l}\text { High expression } \\
\qquad(\mathrm{n}=38)\end{array}$ & $\begin{array}{l}\text { Low expression } \\
\quad(n=42)\end{array}$ & P-value & $\begin{array}{l}\text { High expression } \\
\qquad(\mathrm{n}=33)\end{array}$ & $\begin{array}{l}\text { Low expression } \\
\quad(n=47)\end{array}$ & P-value \\
\hline \multicolumn{8}{|l|}{ Sex } \\
\hline Male & 63 & 30 & 33 & 0.967 & 24 & 39 & 0.270 \\
\hline Female & 17 & 8 & 9 & & 9 & 8 & \\
\hline \multicolumn{8}{|l|}{ Age (years) } \\
\hline$<60$ & 46 & 24 & 22 & 0.330 & 20 & 26 & 0.638 \\
\hline$\geq 60$ & 34 & 14 & 20 & & 13 & 21 & \\
\hline \multicolumn{8}{|c|}{$\begin{array}{l}\text { Size of primary } \\
\text { tumor }(\mathrm{cm})\end{array}$} \\
\hline$<5$ & 49 & 21 & 28 & 0.296 & 22 & 27 & 0.405 \\
\hline$\geq 5$ & 31 & 17 & 14 & & 11 & 20 & \\
\hline \multicolumn{8}{|c|}{ Vascular invasion } \\
\hline Present & 30 & 20 & 10 & 0.008 & 16 & 14 & 0.089 \\
\hline Absent & 50 & 18 & 32 & & 17 & 33 & \\
\hline \multicolumn{8}{|c|}{$\begin{array}{l}\text { Intrahepatic } \\
\text { metastasis }\end{array}$} \\
\hline Present & 22 & 14 & 8 & 0.075 & 15 & 7 & 0.003 \\
\hline Absent & 58 & 24 & 34 & & 18 & 40 & \\
\hline \multicolumn{8}{|c|}{ Serum AFP (ng/ml) } \\
\hline$<200$ & 48 & 25 & 23 & 0.315 & 18 & 30 & 0.404 \\
\hline$\geq 200$ & 32 & 13 & 19 & & 15 & 17 & \\
\hline \multicolumn{8}{|c|}{ Liver cirrhosis } \\
\hline Present & 38 & 16 & 22 & 0.358 & 13 & 25 & 0.224 \\
\hline Absent & 42 & 22 & 20 & & 20 & 22 & \\
\hline \multicolumn{8}{|c|}{ Differentiation } \\
\hline Well & 14 & 6 & 8 & 0.630 & 7 & 7 & 0.746 \\
\hline Moderate & 55 & 28 & 27 & & 22 & 33 & \\
\hline Poor & 11 & 4 & 7 & & 4 & 7 & \\
\hline \multicolumn{8}{|l|}{ TNM stage } \\
\hline I-II & 43 & 14 & 29 & 0.004 & 13 & 30 & 0.031 \\
\hline III-IV & 37 & 24 & 13 & & 20 & 17 & \\
\hline
\end{tabular}

ZEB1, zinc finger E-box-binding homeobox 1; VIM, vimentin; AFP, $\alpha$-fetoprotein; TNM, tumor-node-metastasis.

VIM forward, 5'-AAAACACCCTGCAATCTTTCAGA-3', and reverse, 5'-GATTCCACTTTGCGTTCAAGGT-3'; $\beta$-actin forward, 5'-AGTTGCGTTACACCCTTTCTTGAC-3', and reverse, 5'-GCTCGCTCCAACCGACTGC-3'. The comparative quantification cycle (Cq) method (14) was applied to quantify the expression levels of mRNA. The relative quantity was calculated using the equation $2^{-\Delta \mathrm{Cq}}$ method where $\Delta \mathrm{Cq}=$ $\left(\mathrm{Cq}_{\mathrm{mRNA}}\right.$ of interest $\left.\mathrm{Cq}_{\beta \text {-actin }}\right)$. For the conventional PCR, the relative amount was represented as the grayscale ratio of mRNA of interest to $\beta$-actin.

ZEB1 small interfering (si)RNA and cell transfection. ZEB1 siRNA (siRNA-ZEB1) and negative control siRNA (siRNA-NC) were purchased from Santa Cruz Biotechnology, Inc. and were transfected into Huh-7 cells during the logarithmic growth phase using Lipofectamine 2000 liposome (Invitrogen; Thermo Fisher Scientific, Inc.) following the manufacturer's protocol. The cells were incubated with the siRNA transfection complex for $12 \mathrm{~h}$ at $37^{\circ} \mathrm{C}$, and were then harvested for mRNA and protein expression changes, which were assayed via RT-qPCR and western blot analyses, at $12 \mathrm{~h}$.

In vitro cell proliferation assay. Using a 96-well plate, a total of $8 \times 10^{3}$ Huh-7 cells were seeded into each well and incubated for $18 \mathrm{~h}$. WST-8 $(10 \mu \mathrm{l})$ from the Cell Counting kit-8 (CCK-8; Boster Biological Technology, Wuhan, China) was then added to each well. Following incubation at $37^{\circ} \mathrm{C}$ in $5 \% \mathrm{CO}_{2}$ for 0.5 , $1,2,3$ and $4 \mathrm{~h}$, the absorbance of each sample was measured at a wavelength of $450 \mathrm{~nm}$. 
Table II. Primers used in the present study.

Name

Sequence (5'-3')

EcorI-VIM-promoterFor

VIM-mut1-promoterRev

VIM-mut1-promoterFor

BamHI-VIM-promoterRev

VIM-mut2-promoterRev

VIM-mut2-promoterFor

VIM-promoterFor

VIM-promoterRev
cggaattcAGATCTTGTTTAAAAAGTTGGGT

GTCCGCCCCAGACCCGCGGGCAAAGGAGCGGGAAG

TGCCCGCGGGTCTGGGGCGGACACCGCAGCCCCGAGACCGCC cgcggatccGGCTGCGGAGGGTGGCGATGGCCT

GCCCACCCCTGGGGGCGCCCTCGAGCCTT

GAGGGCGCCCCCAGGGGTGGGCCCCACCCTCCCCGCTTCTCGCT

AGTTACTTAAGCTCGGGCCC

TTGTTCTCGGTGGGCTTGGC

VIM, vimentin.

Table III. Results of prediction.

\begin{tabular}{|c|c|c|c|c|c|c|c|}
\hline Model ID & $\begin{array}{l}\text { Model } \\
\text { name }\end{array}$ & Score & Relative score & Start & End & Strand & $\begin{array}{c}\text { Predicted } \\
\text { site sequence }\end{array}$ \\
\hline MA0103.2 & ZEB1 & 4.883 & 0.828309335350541 & 768 & 776 & 1 & CCCCGCCTG \\
\hline MA0103.2 & ZEB1 & 4.883 & 0.828309335350541 & 1,023 & 1,031 & 1 & CCCCACCCG \\
\hline
\end{tabular}

ZEB1, zinc finger E-box-binding homeobox 1.

Bromodeoxyuridine (BrdU)-labeling assay. The Huh-7 cells were seeded on a slide and incubated in culture medium with or without siRNA-ZEB1 transfection for 1 day. BrdU (10 $\mu \mathrm{M}$; Sigma-Aldrich; Merck KgaA, Darmstadt, Germany) was added to the culture for $1 \mathrm{~h}$. The cells were fixed with $4 \%$ paraformaldehyde and were then exposed to ice-cold methanol for $2 \mathrm{~min}$, incubated in freshly prepared $2 \mathrm{M} \mathrm{HCl}$ for $1 \mathrm{~h}$ at room temperature, and then incubated in $0.1 \mathrm{M}$ sodium borate for 2 min. Finally, immunocytochemistry for the BrdU-labeling of proliferative Huh-7 cells was performed, and images were captured using a Zeiss epifluorescence microscope with a charge-coupled device camera and processed using Axiovert software (v 4.9.1; both Zeiss AG, Oberkochen, Germany).

In vitro invasion assay. Transwell chambers precoated with Matrigel (BD Biosciences, San Jose, CA, USA) were used to perform the invasion assay. The Huh-7 cells were cultured in serum-free medium in the upper chambers of a Transwell plate (Corning Inc., Corning, NY, USA), separated from the lower chambers with permeable $8.0-\mu \mathrm{m}$ polycarbonate membranes. Medium supplemented with $10 \%$ fetal bovine serum was added into the lower chambers as a chemoattractant. After $24 \mathrm{~h}$ of incubation at $37^{\circ} \mathrm{C}$, those cells which had invaded through the membrane were fixed with $75 \%$ ethanol and stained with $0.1 \%$ crystal violet solution. The numbers of stained cells were manually counted under an inverted microscope in five different fields of each filter. At least three independent experiments were performed.

In vitro migration assay. The Huh-7 cells were cultured in 6 -well plates (seeded at a density of $1 \times 10^{6}$ cells/well). A wound was created in the confluent cell monolayer using a sterile $10-\mu 1$ pipette tip and incubated in serum-free medium for $60 \mathrm{~h}$. The migration of cells into the wound and recovery of the monolayer were assessed and images were captured every $12 \mathrm{~h}$ up to $60 \mathrm{~h}$ using a phase contrast microscope.

Construction of recombinant plasmid. The section containing the VIM promoter region was obtained by PCR as aforementioned using VIM-promoter forward and VIM-promoter reverse primers (Table II). The putative binding sites for ZEB1 were predicted using the JASPAR database (http://jaspar. genereg.net/) and the results are shown in Table III. The primers were designed to amplify these predicted sites, and the restriction sites for EcoRI and BamHI were added into these sequences (Table II). The thermocycling conditions comprised denaturation at $95^{\circ} \mathrm{C}$ for $10 \mathrm{~min}$, followed by 40 cycles of denaturation at $95^{\circ} \mathrm{C}$ for $30 \mathrm{sec}$, annealing at $60^{\circ} \mathrm{C}$ for $30 \mathrm{sec}$ and extension at $72^{\circ} \mathrm{C}$ for $30 \mathrm{sec}$. The PCR products were digested by $E c o$ RI and $B a m H I$ and ligated into the pEZX-PG02 vector (GeneCopoeia, Inc., Rockville, MD, USA) using T4 DNA ligase (Takara Bio, Inc.). Subsequently, E. coli $\mathrm{DH} 5 \alpha$ cells were transformed with these products and cultured on Luria-Bertani medium containing kanamycin (all Cwbio Biotechnology, Inc., Beijing, China), and the plates were incubated at $37^{\circ} \mathrm{C}$ for $20 \mathrm{~h}$. Individual colonies were screened and grown in the kanamycin-containing liquid medium overnight. Subsequently, the plasmids were extracted, followed by purification and further sequencing of the inserted sequences. A QuikChange Multi Site-Directed Mutagenesis kit (Stratagene; Agilent Technologies, Inc., La Jolla, CA, USA) was utilized to construct the mutant 

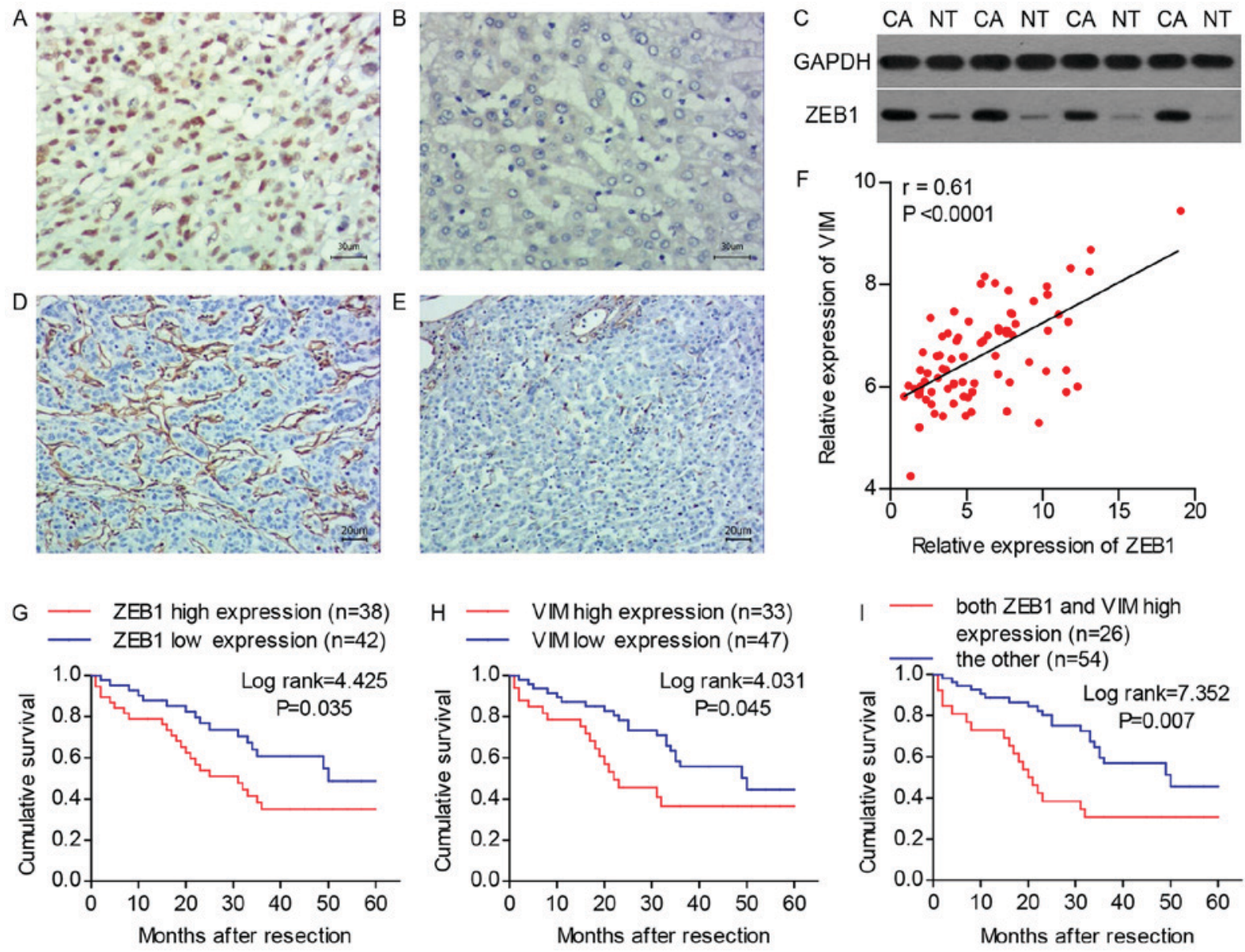

Figure 1. Expression of ZEB1 and VIM in HCC specimens. Immunohistochemical analysis of the protein expression of ZEB1 in (A) HCC tissues and (B) adjacent normal tissues. (C) Western blot analysis of the protein expression of ZEB1 in HCC tissues and adjacent normal tissues. (D) Increased expression of VIM in the extracellular matrix of HCC tissues. (E) Reduced expression of VIM in the adjacent normal tissues. (F) Scatter plots of ZEB1 vs. VIM expression. Pearson's correlation coefficients (r) and P-values are shown. (G) A high expression of ZEB1 was significantly associated with poor overall survival (months) compared with a low expression of ZEB1. (H) A high expression of VIM was significantly associated with poor overall survival (months) compared with low VIM expression. (I) Compared with other expression pattern combinations, the high expression of both ZEB1 and VIM was significantly associated with poorer overall survival (months). ZEB1, zinc finger E-box-binding homeobox 1; VIM, vimentin; HCC, hepatocellular carcinoma; CA, HCC tissues; NT, normal tissues.

pEZX-PG02-VIM promoter plasmids prior to the sequencing analysis.

Plasmid transfection and luciferase reporter assay. The Huh-7 cells were seeded on 48 -well plates $\left(5 \times 10^{4}\right.$ cells $\left./ \mathrm{ml}\right)$. After $24 \mathrm{~h}$, the cells were treated with Opti-MEM reduced serum medium (Invitrogen; Thermo Fisher Scientific, Inc.) in preparation for transfection. Lipofectamine 2000 reagent was used according to the manufacturer's protocol. After $48 \mathrm{~h}$, the cells were harvested for luciferase detection using the GLuc Assay kit (GeneCopoeia, Inc.). All values were obtained from at least three independent repetitions of the transfection.

Statistical analysis. Continuous variables were compared using the Kruskal-Wallis test and categorical data were compared using the $\chi^{2}$ or Fisher's exact tests. The Kaplan-Meier method was used for survival analysis, and differences in survival were estimated using the log-rank test. Multivariate analysis of prognostic factors for survival was performed using a Cox logistic regression model. All statistical analyses were performed with GraphPad Prism 5.0 software. $\mathrm{P}<0.05$ was considered to indicate a statistically significant difference.

\section{Results}

Expression of ZEBI and VIM in HCC. The results of the immunohistochemistry revealed that the protein expression level of the ZEB1 was higher in the tumor tissues compared with that in the adjacent normal tissues and was primarily localized in the nucleus (Fig. 1A and B). This result was confirmed by western blot analysis (Fig. 1C). According to the expression level of ZEB1, the HCC tissue specimens were divided into four groups: Absent $(n=42), 1-5 \%(n=25), 6-10 \%$ $(n=10)$ and $>10 \%(n=3)$. The expression of ZEB1 was defined as high if at least $1 \%$ of HCC cells exhibited nuclear staining $(\mathrm{n}=38 ; 47.5 \%)$, or as low if there were no stained HCC cells $(n=42 ; 52.5 \%)$. The expression of VIM was detected in the extracellular matrix and categorized by comparing its level in HCC tissues with that in adjacent normal tissues. The HCC tissues with a higher staining intensity compared with that of adjacent normal tissues were deemed to have high expression ( $n=33 ; 41.3 \%$; Fig. 1D), whereas those with a staining intensity equal to or weaker than that in the adjacent normal tissues were deemed to have low expression $(n=47$; $58.7 \%$; Fig. 1E). The RT-qPCR analysis demonstrated that 

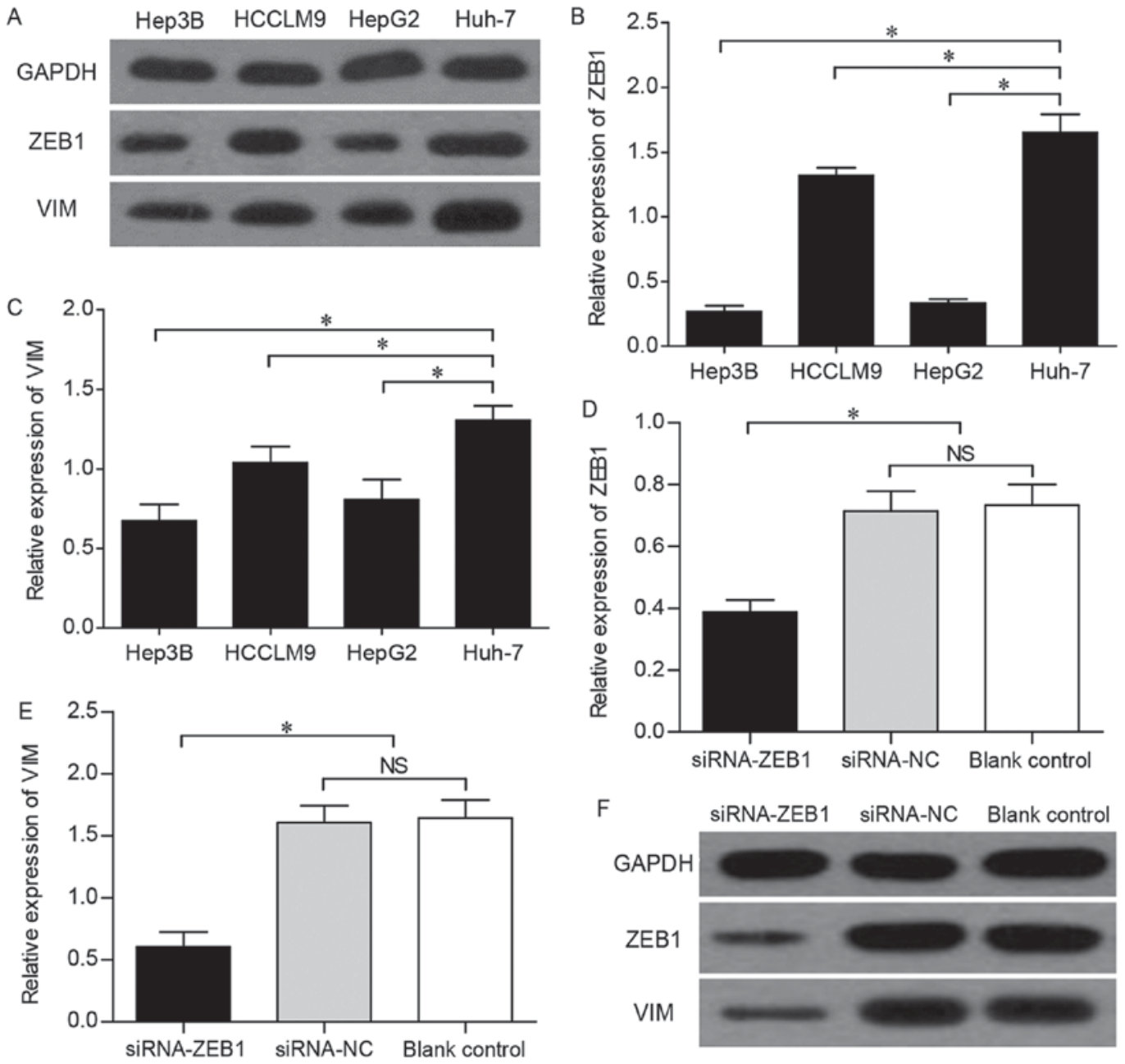

Figure 2. Effects of ZEB1 interference on the expression of VIM. (A) Western blot analysis of the protein expression of ZEB1 and VIM in the Hep3B, HCCLM9, HepG2 and Huh-7 liver cancer cell lines. Expression of (B) ZEB1 and (C) VIM in the liver cancer cell lines was investigated using reverse transcription-quantitative polymerase chain reaction analysis. Expression of (D) ZEB1 and (E) VIM in Huh-7 cells transfected with siRNA-ZEB1. (F) Western blot analysis of the protein expression of ZEB1 and VIM in Huh-7 cells transfected with siRNA-ZEB1. "P<0.05. ZEB1, zinc finger E-box-binding homeobox 1; VIM, vimentin; siRNA, small interfering RNA; NC, negative control; NS, not significant.

the expression of ZEB1 was significantly correlated with the expression of VIM (Fig. 1F). The correlations between immunohistochemical expression and clinicopathological variables are shown in Table I. A high expression of ZEB1 was significantly associated with vascular invasion $(\mathrm{P}=0.008)$ and TNM stage $(\mathrm{P}=0.004)$. Similarly, a high expression of VIM was significantly correlated with intrahepatic metastasis $(\mathrm{P}=0.003)$ and $\mathrm{TNM}$ stage $(\mathrm{P}=0.031)$. The overall survival rates following surgery based on the expression of ZEB1 and VIM are shown in Fig. $1 \mathrm{G}$ and H. Kaplan-Meier analysis demonstrated that a high expression of ZEB1 was significantly associated with a poor overall survival rate $(\log$ rank $=4.425$; $\mathrm{P}=0.035)$ and the overall survival rate was significantly higher in the low-VIM expression group compared with that in the high-VIM expression group (log rank=4.031; $\mathrm{P}=0.045)$. In addition, a high expression of both ZEB1 and VIM was compared with other expression pattern combinations, and the former group exhibited a significantly poorer overall survival rate $(\log$ rank=7.352; $\mathrm{P}=0.007 ;$ Fig. 1I).

Protein and mRNA expression of VIM and of ZEBI in different liver cancer cell lines. The western blot analysis revealed that ZEB1 and VIM exhibited the highest expression levels in Huh-7 cells (Fig. 2A). In addition, the RT-qPCR analysis revealed that Huh-7 cells expressed significantly higher levels of ZEB1 and VIM compared with other HCC cell lines (Fig. 2B and C). These data confirmed that the expression trend of VIM was consistent with that of ZEB1 in different liver cancer cell lines.

Effects of ZEBI interference on the expression of VIM. To assess the possible effect of ZEB1 on the expression of VIM, siRNAs were used to knockdown ZEB1 in Huh-7 cells, in which the expression of ZEB1 was found to be high, as mentioned above. The Huh-7 cells were divided into three groups: The siRNA-ZEB1 transfected group, the siRNA-NC group and the blank control group. The knockdown of ZEB1 was confirmed by conventional PCR, which revealed that, compared with that in the siRNA-NC and the blank control groups, the mRNA expression level of ZEB1 in the Huh-7 cells of the siRNA-ZEB1-transfected group was significantly decreased $(\mathrm{P}<0.05$; Fig. $2 \mathrm{D})$, indicating that the interference effect of siRNA on ZEB1 was effective and specific. Subsequently, the siRNA-ZEB1-transfected group was found 


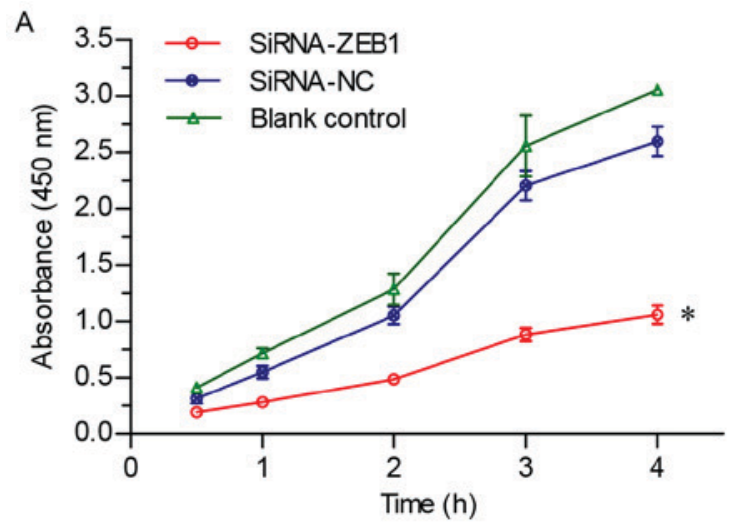

C

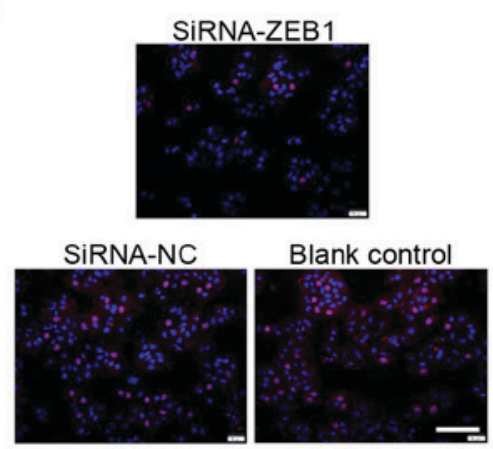

F
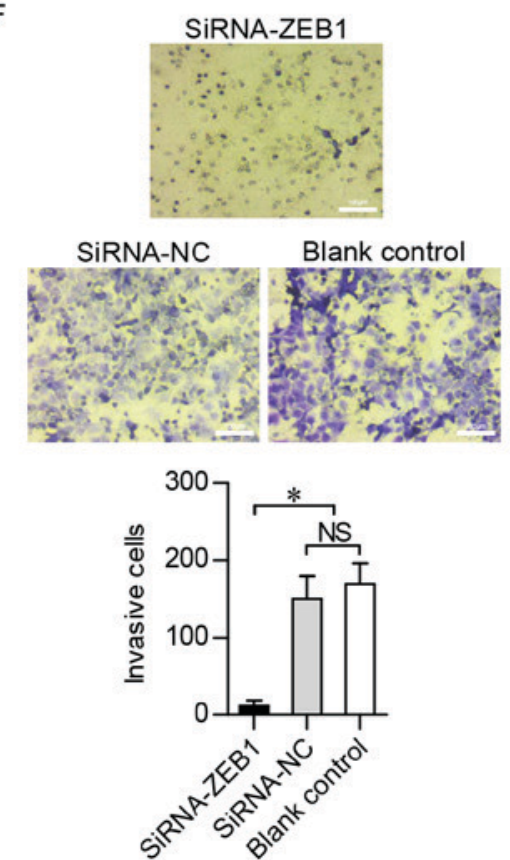

D

G
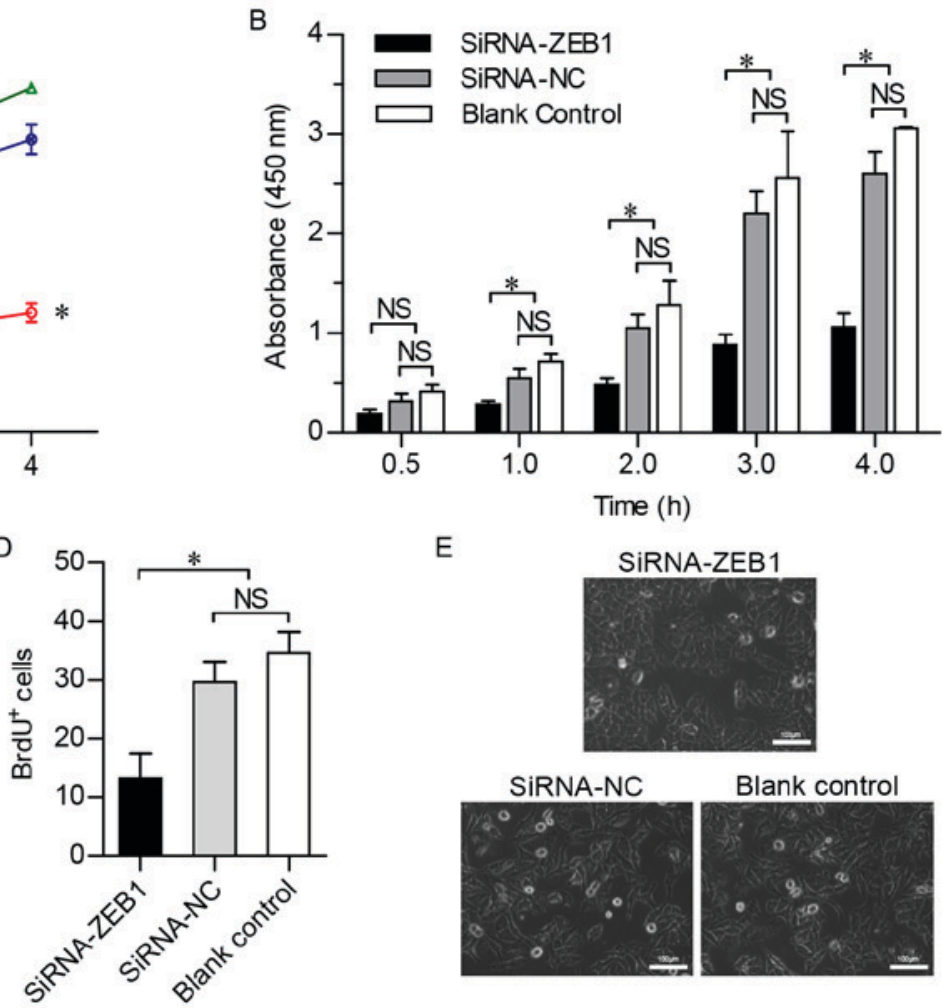

E
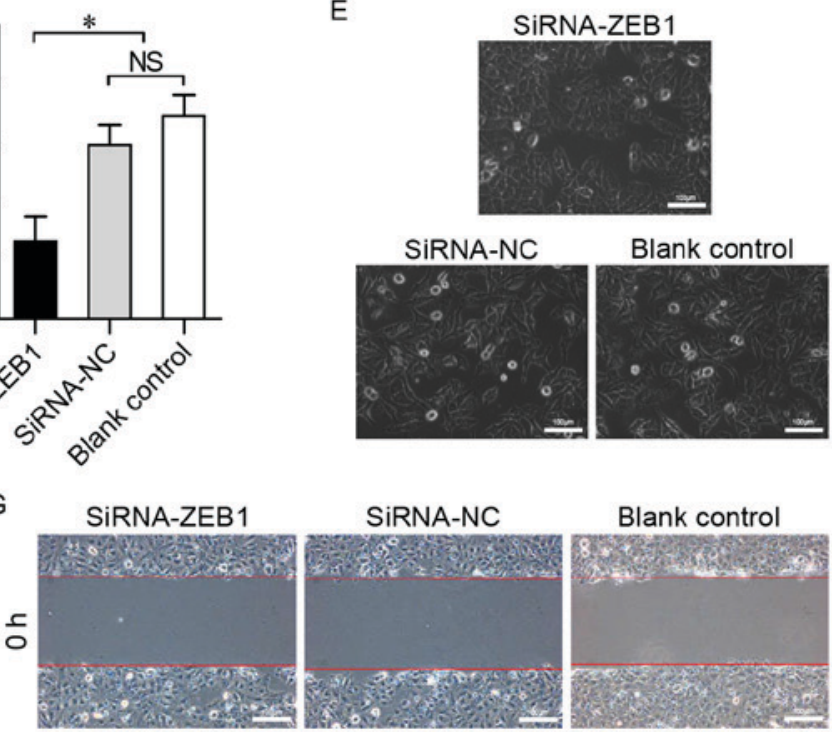

SiRNA-NC

Blank control
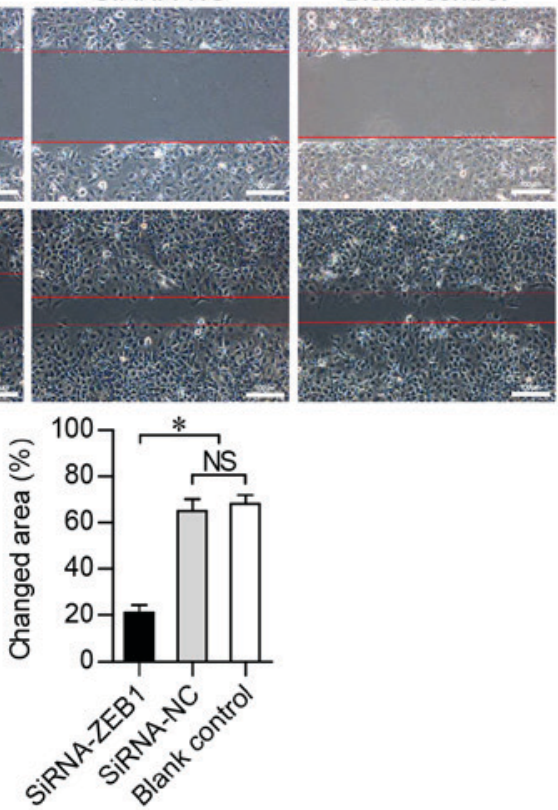

Figure 3. Effects of ZEB1 interference on cell proliferation, cell cycle, invasion and migration in vitro. (A) Cell Counting kit-8 assays of Huh-7 cells transfected with siRNA-ZEB1. (B) Statistical analysis of absorbance in different groups. (C) BrdU-uptake assay of Huh-7 cells transfected with siRNA-ZEB1. Scale bar=100 $\mu \mathrm{m}$. (D) Results of BrD uptake. (E) Inverted micrographs of indicated Huh-7 cells. Scale bar=100 $\mu \mathrm{m}$. (F) Crystal violet staining of Huh-7 cells that had invaded through the polycarbonate membrane of the Transwell chamber. Scale bar $=100 \mu \mathrm{m}$. (G) Cell migration was assessed using a wound-healing assay. The residual wound was markedly wider in the siRNA-ZEB1-transfected group compared with that in the siRNA-NC and the blank control groups at $48 \mathrm{~h}$. Scale bar=100 $\mu \mathrm{m}$. ${ }^{*} \mathrm{P}<0.05$. ZEB1, zinc finger E-box-binding homeobox 1; siRNA, small interfering RNA; NC, negative control; BrdU, bromodeoxyuridine; NS, not significant.

to be associated with a significantly lower mRNA expression of VIM compared with that in the two other groups $(\mathrm{P}<0.05$; Fig. 2E). Furthermore, western blot analysis demonstrated that the successful interference led to marked downregulation of the expression of ZEB1 and VIM ( $\mathrm{P}<0.05$; Fig. 2F), suggesting that the expression of VIM was subject to regulation by ZEB1.
Effects of ZEB1 interference on cell proliferation in vitro. To investigate the effect of the suppression of ZEB1 on cell viability, a CCK-8 assay was used over a period of $4 \mathrm{~h}$, and cell viability was found to be significantly lower in the siRNA-ZEB1-transfected group compared with that in the other two groups $(\mathrm{P}<0.05$; Fig. $3 \mathrm{~A}$ and $\mathrm{B})$, indicating that cell 
Table IV. Luciferase activity of the co-transfection groups.

Luciferase expression (AU)

\begin{tabular}{lrrr} 
Group & \multicolumn{1}{c}{ Test 1} & Test 2 & Test 3 \\
\hline siRNA-ZEB1+PG02-VIM & 707,230 & 753,450 & 794,160 \\
siRNA-NC+PG02-VIM & $1,320,010$ & $1,370,250$ & $1,344,110$ \\
siRNA-ZEB1+PG02-VIM-mut1 & $18,51,070$ & $1,894,100$ & $1,803,880$ \\
siRNA-NC+PG02-VIM-mut1 & $2,454,300$ & $2,401,670$ & $2,495,070$ \\
siRNA-ZEB1+PG02-VIM-mut2 & 833,960 & 836,770 & 774,770 \\
siRNA-NC+PG02-VIM-mut2 & 877,170 & 897,260 & 831,260 \\
\hline
\end{tabular}

siRNA, small interfering RNA; ZEB1, zinc finger E-box-binding homeobox 1; VIM, vimentin; NC, negative control.
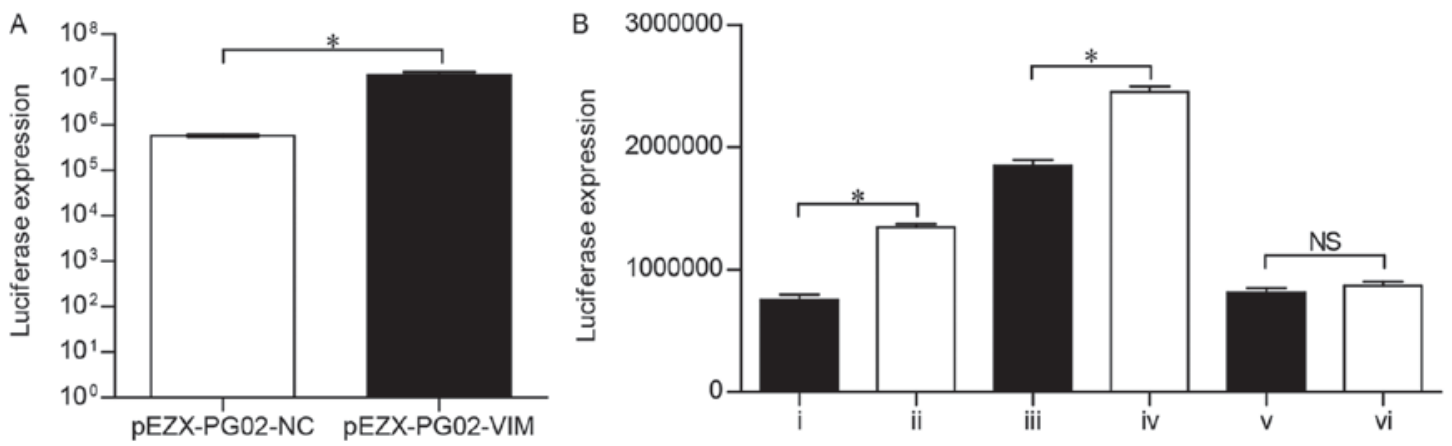

Figure 4. Effects of ZEB1 interference on VIM promoter activity. (A) Luciferase activity in Huh-7 cells with the recombinant plasmid (pEZX-PG02-VIM) compared with the control group (pEZX-PG02-NC). (B) Huh-7 cells were transfected with the indicated combinations of vectors and the luciferase activity was measured to evaluate the promoter activity. i) siRNA-ZEB1+pEZX-PG02-VIM; ii) siRNA-NC+pEZX-PG02-VIM; iii) siRNA-ZEB1+pEZX-PG02-VIM-mut1; iv) siRNA-NC+pEZX-PG02-VIM-mut1; v) siRNA-ZEB1+pEZX-PG02-VIM-mut2; vi) siRNA-NC+pEZX-PG02-VIM-mut2. "P<0.05. ZEB1, zinc finger E-box-binding homeobox 1; VIM, vimentin; siRNA, small interfering RNA; NC, negative control; NS, not significant.

viability was suppressed by the knockdown of ZEB1. In addition, the BrdU-labeling assay revealed that DNA synthesis was inhibited in the siRNA-ZEB1-transfected Huh-7 cells $(\mathrm{P}<0.05$; Fig. 3C and D). These results confirmed that cell proliferation was markedly inhibited by silencing ZEB1.

Effects of ZEBI interference on invasion and migration in vitro. The Huh-7 cells transfected with siRNA-ZEB1 had an oval shape (Fig. 3E), suggesting that the knockdown of ZEB1 prevented the HCC cells from undergoing EMT. To further verify whether ZEB1 inhibited the invasion and migration of HCC cells, the in vitro invasiveness of Huh-7 cells was detected using the Transwell invasion chamber assay. Crystal violet staining revealed that the number of Huh-7 cells invading through the polycarbonate membrane of the Transwell invasion chamber was significantly lower in the siRNA-ZEB1-transfected group $(\mathrm{P}<0.05)$. No significant difference in invasiveness was found between the siRNA-NC and the blank control groups (Fig. 3F). In addition, wound-healing assays were performed to investigate the cell migration ability in the three groups, revealing that the migration of the siRNA-ZEB1-transfected cells was significantly slower compared with that in the other two groups at $48 \mathrm{~h}$ post-wounding (Fig. 3G). These results demonstrated that downregulation of the ZEB1 gene suppressed the motility of Huh-7 cells.
Construction and identification of the recombinant plasmid. According to the results of prediction (Table III), two types of mutational pEZX-PG02-VIM promoter plasmids (pEZX-PG02-VIM-mut1 and pEZX-PG02-VIM-mut2) were constructed. To verify the activity of the pEZX-PG02-VIM promoter vector, the recombinant plasmid was transfected into Huh-7 cells and the luciferase expression was detected using a fluorescent microscope. Compared with the pEZX-PG02-NC group, the cells containing the pEZX-PG02-VIM recombinant plasmid exhibited significantly higher luciferase activity $(\mathrm{P}<0.05$; Fig. 4A), indicating that the VIM promoter sequence can be bound and activated in Huh-7 cells.

Effects of ZEBI interference on VIM promoter activity. To confirm that ZEB1 has the ability to regulate the expression of VIM and simultaneously identify the binding sites in the VIM promoter sequence, six categories of co-transfection were conducted: i) siRNA-ZEB1+pEZX-PG02-VIM; ii) siRNA-NC+pEZX-PG02-VIM; iii) siRNA-ZEB1+pEZX-PG 02-VIM-mut1; iv) siRNA-NC+pEZX-PG02-VIM-mut1; v) siRN A-ZEB1+pEZX-PG02-VIM-mut2; vi) siRNA-NC+pEZX-PG02 -VIM-mut2; subsequently, the luciferase expression was detected three times in each group (Table IV). The luciferase activity in the siRNA-ZEB1+pEZX-PG02-VIM group was significantly lower than that in the siRNA-NC+pEZX-PG02-VIM group $(\mathrm{P}<0.0001$; Fig. 4B), which demonstrated that, when the Huh-7 
cells were transfected with siRNA-ZEB1, the VIM promoter activity markedly decreased. Therefore, the downregulation of ZEB1 markedly reduced the transcription of the VIM gene. Compared with the siRNA-ZEB1+pEZX-PG02-VIM-mut1 group, the siRNA-NC+pEZX-PG02-VIM-mut1 group was associated with significantly higher luciferase activity $(\mathrm{P}<0.0001$; Fig. 4B). More specifically, ZEB1 was able to regulate the VIM-mut1 promoter activity. However, the results revealed no statistically significant difference in luciferase activity between the siRNA-ZEB1+pEZX-PG02-VIM-mut2 and siRNA-NC+pEZX-PG02-VIM-mut2 groups ( $\mathrm{P}>0.05$; Fig. 4B), indicating that ZEB1 did not lead to activation of the VIM-mut2 promoter.

\section{Discussion}

EMT is considered to be a crucial step in tumor aggressiveness, oncogenic progression and cancer metastasis (15). ZEB1 has been identified to serve a decisive role in the induction of EMT and enhancement of the invasive and migratory phenotype in various tumor cell lines (16-18). The present study investigated the role of ZEB1 in HCC and reported a correlation between ZEB1 and VIM. The results of immunohistochemistry, western blotting, cell proliferation, invasion and migration assays in vitro revealed that a high expression of ZEB1 was significantly associated with the malignant progression of HCC. Simultaneously, ZEB1 silencing led to attenuation of the malignant biological behavior of HCC cells. Therefore, it may be concluded that the expression of ZEB1 is closely associated with tumorigenesis and it may be a biomarker for the malignant phenotype of HCC, which is of considerable value in the monitoring of tumor progression, treatment evaluation and prediction of prognosis. With regard to its clinical application, a series of scientific and clinical trials is warranted for the assessment of this biomarker. Few studies have investigated the association between the expression of ZEB1 and VIM, and the specific regulatory relationship remains to be elucidated at present. The present study demonstrated a significant positive correlation between the expression of ZEB1 and VIM in the immunohistochemical analysis. Subsequently, the expression levels of ZEB1 and VIM were detected in liver cancer cell lines, including three HCC cell lines (Hep3B, HCCLM9 and Huh-7) and a hepatoblastoma cell line (HepG2). It was observed that the expression trend of VIM was entirely consistent with that of ZEB1 in the liver cancer cell lines at the mRNA and protein levels. As ZEB1 and VIM exhibited the highest expression levels in Huh-7 HCC cells, the subsequent experiments were performed using Huh-7 cells. Mechanistically, a significant downregulation of VIM was observed following the successful silencing of ZEB1, which indicated that the expression of VIM was strictly subject to regulation by ZEB1. A promoter analysis assay was conducted to investigate and identify the specific binding site. The luciferase reporter assay is one of the key techniques for investigating the regulation of gene expression in mammalian cell cultures (19). More specifically, the transcriptional activity can be evaluated by detecting the luciferase activity of recombinant plasmid-transfected cells. Based on the prediction of binding sites in the JASPAR database, two mutational pEZX-PG02-VIM promoter plasmids, which contained different mutation sites, were designed and constructed. The results of the luciferase assay demonstrated that the transcriptional activity of VIM was affected by ZEB1 at the gene expression level. In addition, the results of subsequent experiments suggested that ZEB1 was able to regulate VIM-mut1 promoter activity, but did not lead to activation of the VIM-mut 2 promoter. The putative binding site sequence for ZEB1 in the VIM promoter was found to be CCCCACCCG (1023-1031). Therefore, ZEB1 was considered to preferentially bind to the second putative site (1023-1031) of the VIM promoter (Table III) to regulate the transcriptional activity of VIM.

In conclusion, the present study demonstrated that ZEB1 and VIM were concomitantly upregulated in HCC tissues, and ZEB1 was crucial in the tumorigenesis and metastasis of HCC. Finally, the data revealed that ZEB1 was able to bind to and regulate the transcription of the VIM gene, suggesting that one of the mechanisms through which ZEB1 induces tumorigenesis is regulation of the expression of VIM.

\section{Acknowledgements}

Not applicable.

\section{Funding}

No funding was received.

\section{Availability of data and materials}

All the datasets generated and analyzed in the present study are included in this published article.

\section{Authors' contributions}

YQ performed all experiments. JY performed all data analysis. MZ collected clinical samples. FQ provided experimental support for IHC and luciferase report assays. XL designed the project. JY contributed to manuscript revision. All authors have read and approved the final version of this manuscript.

\section{Ethics approval and consent to participate}

All patients provided written informed consent to participate in the present study, and the study protocol was approved by the Ethics Committee of The Central Hospital of Enshi Autonomous Prefecture.

\section{Patient consent for publication}

Not applicable.

\section{Competing interests}

The authors declare that they have no competing interests.

\section{References}

1. El-Serag HB and Rudolph KL: Hepatocellular carcinoma: Epidemiology and molecular carcinogenesis. Gastroenterology 132: 2557-2576, 2007. 
2. Lau WY and Lai EC: Hepatocellular carcinoma: Current management and recent advances. Hepatobiliary Pancreat Dis Int 7: 237-257, 2008.

3. Kalluri R and Weinberg RA: The basics of epithelial-mesenchymal transition. J Clin Invest 119: 1420-1428, 2009.

4. Iwatsuki M, Mimori K, Yokobori T, Ishi H, Beppu T, Nakamori S, Baba $\mathrm{H}$ and Mori M: Epithelial-mesenchymal transition in cancer development and its clinical significance. Cancer Sci 101: 293-299, 2010

5. Remacle JE, Kraft H, Lerchner W, Wuytens G, Collart C, Verschueren K, Smith JC and Huylebroeck D: New mode of DNA binding of multi-zinc finger transcription factors: DeltaEF1 family members bind with two hands to two target sites. EMBO J 18: 5073-5084, 1999.

6. Postigo AA, Depp JL, Taylor JJ and Kroll KL: Regulation of Smad signaling through a differential recruitment of coactivators and corepressors by ZEB proteins. EMBO J 22: 2453-2462, 2003.

7. Singh M, Spoelstra NS, Jean A, Howe E, Torkko KC, Clark HR, Darling DS, Shroyer KR, Horwitz KB, Broaddus RR and Richer JK: ZEB1 expression in type I vs type II endometrial cancers: A marker of aggressive disease. Mod Pathol 21: 912-923, 2008.

8. Sanchez-Tillo E, de Barrios O, Siles L, Amendola PG, Darling DS, Cuatrecasas M, Castells A and Postigo A: ZEB1 Promotes invasiveness of colorectal carcinoma cells through the opposing regulation of uPA and PAI-1. Clin Cancer Res 19: 1071-1082, 2013

9. Graham TR, Zhau HE, Odero-Marah VA, Osunkoya AO, Kimbro KS, Tighiouart M, Liu T, Simons JW and O'Regan RM: Insulin-like growth factor-I-dependent up-regulation of ZEB1 drives epithelial-to-mesenchymal transition in human prostate cancer cells. Cancer Res 68: 2479-2488, 2008.

10. Eger A, Aigner K, Sonderegger S, Dampier B, Oehler S, Schreiber M, Berx G, Cano A, Beug H and Foisner R: DeltaEF1 is a transcriptional repressor of E-cadherin and regulates epithelial plasticity in breast cancer cells. Oncogene 24: 2375-2385, 2005.
11. Putzke AP, Ventura AP, Bailey AM, Akture C, Opoku-Ansah J, Celiktaş M, Hwang MS, Darling DS, Coleman IM, Nelson PS, et al: Metastatic progression of prostate cancer and e-cadherin regulation by zeb1 and SRC family kinases. Am J Pathol 179: 400-410, 2011.

12. Lopez-Terrada D, Cheung SW, Finegold MJ and Knowles BB: Hep G2 is a hepatoblastoma-derived cell line. Hum Pathol 40: $1512-1515,2009$.

13. Li Y, Tang ZY, Ye SL, Liu YK, Chen J, Xue Q, Chen J, Gao DM and Bao WH: Establishment of cell clones with different metastatic potential from the metastatic hepatocellular carcinoma cell line MHCC97. World J Gastroenterol 7: 630-636, 2001.

14. Livak KJ and Schmittgen TD: Analysis of relative gene expression data using real-time quantitative PCR and the 2(-Delta Delta C(T)) method. Methods 25: 402-408, 2001.

15. Polyak K and Weinberg RA: Transitions between epithelial and mesenchymal states: Acquisition of malignant and stem cell traits. Nat Rev Cancer 9: 265-273, 2009.

16. Bae GY, Choi SJ, Lee JS, Jo J, Lee J, Kim J and Cha HJ: Loss of E-cadherin activates EGFR-MEK/ERK signaling, which promotes invasion via the ZEB1/MMP2 axis in non-small cell lung cancer. Oncotarget 4: 2512-2522, 2013.

17. Wang Y, Wen M, Kwon Y, Xu Y, Liu Y, Zhang P, He X, Wang Q, Huang Y, Jen KY, et al: CUL4A induces epithelial-mesenchymal transition and promotes cancer metastasis by regulating ZEB1 expression. Cancer Res 74: 520-531, 2014.

18. Siebzehnrubl FA, Silver DJ, Tugertimur B, Deleyrolle LP, Siebzehnrubl D, Sarkisian MR, Devers KG, Yachnis AT, Kupper MD, Neal D, et al: The ZEB1 pathway links glioblastoma initiation, invasion and chemoresistance. EMBO Mol Med 5: 1196-1212, 2013.

19. Yun C and Dasgupta R: Luciferase reporter assay in Drosophila and mammalian tissue culture cells. Curr Protoc Chem Biol 6: 7-23, 2014. 